\title{
EGFR exon 20 insertion mutations and response to osimertinib in non-small-cell lung cancer
}

Wenfeng Fang ${ }^{1 *}$, Yihua Huang ${ }^{1 \dagger}$, Shaodong Hong ${ }^{1 \dagger}$, Zhonghan Zhang $^{1 \dagger}$, Minghui Wang $^{2 \dagger}$, Jiadi Gan ${ }^{1}$, Wenjing Wang ${ }^{3}$, Honglin Guo ${ }^{3}$, Kai Wang ${ }^{3}$ and Li Zhang ${ }^{1 *}$

\begin{abstract}
Background: Epidermal growth factor receptor exon 20 insertion (EGFRex20ins) mutations represent approximately $4-12 \%$ of EGFR mutations and are generally refractory to the 1st and 2nd generation EGFR tyrosine kinase inhibitors (TKIs). Development of effective therapies for patients with EGFRex20ins mutant non-small-cell lung carcinoma (NSCLC) represents a great unmet need. Preclinical models have shown that osimertinib is active in NSCLC harboring EGFRex20ins, while the antitumor activity of osimertinib remains to be evaluated in patients with EGFRex20ins mutations.

Methods: Tumor genotyping was performed in 2316 Chinese NSCLC cases with targeted next generation sequencing (NGS) covering the whole exons of EGFR gene. The frequency and genetic characteristics of EGFRexon20ins mutations were analyzed. Furthermore, six patients with specific EGFRexon20ins mutations and receiving osimertinib $80 \mathrm{mg}$ once daily were retrospectively included to assess the antitumor activity and safety of monotherapy osimertinib.

Results: EGFRex20ins mutations were identified in 4.8\% (53/1095) of EGFR mutant NSCLC and 2.3\% (53/2316) of all NSCLC cases. The most frequently identified EGFRexon20ins is A767_V769dup (17/53,32.1\%). We found that the genetic characteristics of EGFRex20ins mutations in Chinese patients with NSCLC were comparable to those reported in Caucasian patients. Four patients with osimertinib therapy achieved partial response and the rest stable disease. Median progression free survival (PFS) was 6.2 months (95\% confidence interval 5.0-12.9 months; range 4.9-14.6 months). The most common adverse events (AEs) were diarrhea (2/6), pruritis (2/6), stomatitis (1/6) and nausea (1/6). No grade 3 or more AEs were documented.

Conclusions: This study revealed that the genetic characteristics of EGFRex20ins mutations in Chinese patients with NSCLC were comparable to those reported in Caucasian patients. Furthermore, our study firstly demonstrated promising antitumor activity of osimertinib in certain EGFRex20ins mutant advanced NSCLC patients, indicating that osimertinib treatment for EGFRex20ins positive patients deserves further study.
\end{abstract}

Keywords: NSCLC, EGFRex20ins, NGS, Osimertinib

\footnotetext{
*Correspondence: fangwf@sysucc.org.cn; zhangli@sysucc.org.cn

tWenfeng Fang, Yihua Huang, Shaodong Hong, Zhonghan Zhang and Minghui Wang contributed equally to this work.

'Department of Medical Oncology, State Key Laboratory of Oncology in

South China, Collaborative Innovation Center for Cancer Medicine, Sun

Yat-Sen University Cancer Center, Guangzhou 510060, People's Republic of

China

Full list of author information is available at the end of the article
}

(c) The Author(s). 2019 Open Access This article is distributed under the terms of the Creative Commons Attribution 4.0 International License (http://creativecommons.org/licenses/by/4.0/), which permits unrestricted use, distribution, and reproduction in any medium, provided you give appropriate credit to the original author(s) and the source, provide a link to the Creative Commons license, and indicate if changes were made. The Creative Commons Public Domain Dedication waiver (http://creativecommons.org/publicdomain/zero/1.0/) applies to the data made available in this article, unless otherwise stated. 


\section{Background}

During the past decades, the identification of specific genomic aberrations and their corresponding targeted therapies have significantly improved the outcome and quality of life for patients with non-small-cell lung cancer (NSCLC). Epidermal growth factor receptor (EGFR) mutation is the first identified targetable driver mutation that was reported in about 17 and 50\% of lung adenocarcinoma in Caucasians and Asians, respectively [1-3]. The most common cluster of mutations in EGFR gene include inframe deletions around the LeuArgGluAla motif (residues 746-750) of exon 19, and the Leu858Arg (L858R) point mutation in exon 21, each accounting for about $45 \%$ of all EGFR mutations. These mutations are termed classic EGFR mutations and are more common in tumors in women, Asians, never smokers, and those with adenocarcinoma [4-6]. The frequency and distribution of EGFR mutations in patients with different ethnic backgrounds also differ $[7,8]$.

Patients with classic EGFR mutations generally have profound radiographic and clinical response to monotherapy EGFR tyrosine kinase inhibitors (TKIs) [9-12]. However, some unclassical EGFR mutations are associated with poor responses with reversible EGFR TKIs $[13,14]$. Among these are most EGFR exon 20 insertion (EGFRex20ins) mutations reported as far. Exon 20 of EGFR encompasses nucleotides that translate into amino acid at position 762 to 823 . It contains a C-helix (residues 762-766) and the loop following C-helix (residues 767-774), where the insertions could induce ligand-independent EGFR pathway activation and give rise to tumorigenesis [15]. The true frequency of EGFRex20ins mutations within the EGFR mutant lung cancer is inconsistent, contributing to roughly $4-12 \%$ of all EGFR mutations identified [16-18]. In most reports, EGFRex20ins mutations are more common in tumors among neversmokers $[16,18]$. However, the genetic and clinical characteristics of NSCLCs harboring EGFRex20ins mutations in Asian populations remain unknown due to the lack of large comprehensive genomic studies.

Preclinical and clinical studies have shown that most EGFRex20ins (except for few subtypes such as EGFR A763_Y764insFQEA) mutant tumors confer resistance to the 1st and 2nd generation EGFR TKIs because the insertions produce steric hindrance and activate EGFR without saliently decreasing affinity for ATP or enhancing affinity for EGFR TKIs [15, 19-23]. Several clinical studies specifically involving tumors with EGFRex20ins mutations are ongoing, with some of them showing preliminary promising activity $[24,25]$. However, there are still no established molecular targeted drugs for NSCLC patients with EGFRx20ins mutations. Development of more effective therapeutics for these specific patients represents a great unmet need.
Osimertinib is an oral, potent, irreversible EGFR TKI selective for sensitizing EGFR and EGFR T790 M resistance mutations. Preclinical studies have reported that osimertinib was active in EGFRex20ins mutant cell lines and tumor xenografts with a wide therapeutic window [26-29]. However, whether the preclinical activity of osimertinib could translate into clinical effect remains unclear.

Herein, we explored the characteristics of EGFRex20ins, as well as the patterns of co-mutations (mutually exclusive or inclusive) in Chinese NSCLC patients. In addition, we assessed the safety and antitumor activity of osimertinib in six advanced NSCLC patients with various EGFRex20ins mutations.

\section{Methods \\ Patients}

The study included a cohort of patients who were referred to OrigiMed (Shanghai, China) for targeted next generation sequencing (NGS) test in China between August 2016 and July 2018. Patient samples and clinical information including gender, age and histologic subtypes were retrieved at the time of referral. Six patients with EGFRex20ins mutant stage IV NSCLC who were treated with osimertinib $80 \mathrm{mg}$ once daily were included to evaluate the antitumor activity of osimertinib. Data were retrospectively collected from digital medical records. The study was approved by the Institutional Review Board of SYSUCC and written informed consent was obtained for each patient prior to sample collection. Written consents were obtained from parents if patients were under 16 years old.

\section{EGFR ex20ins and co-mutations analysis}

DNA from Formalin-fixed, paraffin-embedded tumor tissue and matched blood samples was extracted. Comprehensive genomic profiling was performed by NGS with a 37 or 450 cancer related gene panel covering the whole exons of EGFR gene at a mean coverage depth of $>800 \mathrm{X}$ (1547 cases with 37 panel, and 769 cases with 450 panel). The genomic alterations including single base substitution, insertions/deletions, copy number variations, as well as gene rearrangement and fusions were assessed. As for six patients treated with osimertinib, genetic status was also determined through NGS prior to osimertinib.

\section{Response evaluation}

All six patients received oral osimertinib $80 \mathrm{mg}$ once daily. Radiological follow-up was performed at the first months then once every 2 months with computed tomography (CT) of the thorax and upper abdomen. Regular cerebral magnetic resonance imaging (MRI) with CT was carried out once any patient was confirmed brain 
metastasis. Response was assessed according to Response Criteria in Solid Tumors (RECIST) 1.1 [30]. Progression-free survival (PFS) was defined as the interval from the date of initiation of osimertinib therapy to the date of disease progression or death from any cause, whichever occurred first.

\section{Statistical analyses}

The statistical analyses were performed using the SPSS 20.0 (Chicago, IL, USA). The difference in the frequency of each group was analyzed by the Chi-square test or Fisher's exact test. The median age between groups was compared using nonparametric test. A two-sided $p$ value $<0.05$ was considered statistically significant.

\section{Results}

Frequency and genetic characteristics of EGFRex20ins mutations

Among the 2316 unselective NSCLC tumors, EGFR mutations were identified in 1095 cases (47.3\%). EGFRex20ins mutations were detected in 53 cases, contributing $2.3 \%$ of all NSCLC cases and $4.8 \%$ of EGFR-mutant tumors. Compared with Foundation Medicine (FM) data representing the largest EGFRex20ins cohort, we found that although EGFR mutations were much more common in our Chinese NSCLC patients than that of Western population (47.3\% vs $15.5 \%$ in FM), EGFRex20ins mutation represented a much smaller group in EGFR mutant NSCLC $(4.8 \%$ vs $11.7 \%$ in FM, $p<0.001)$ [17]. Of note, our result was comparable with FM cohort in frequency of EGFRex20ins in total NSCLC (2.3\% vs. $1.8 \%$ in FM, $p=0.12$, Fig. 1 ). The smaller proportion of EGFRex20ins in Chinese populations is due to the larger scale of EGFR mutations than western groups.

The demographic and clinical characteristics of these patients are summarized in Tables 1 and 2. Of the patients with EGFR mutations, EGFRex20ins ranked the fourth most common type, following EGFR exon 19 deletions (436/1095, 39.8\%), L858R (410/1095, 37.4\%) and T790 M mutations (58/1095, 5.3\%) (Fig. 2a). The majority of EGFRex20ins mutations were identified in lung adenocarcinoma $(92.5 \%, 49 / 53)$. EGFRex20ins were also detected in two adenosquamous cases and two NSCLC not otherwise specified (NOS). Median age of patients with exon 20 insertion is 57 (31-85) years.

In total, 20 different variants of exon EGFRex20ins were identified in 53 NSCLC patients. The most frequent variant is A767_V769dup (32.1\%, 17/53), followed by P772_H773dup (4/53, 7.5\%), S768_D770dup (4/53, 7.5\%), N771_H773dup (4/53, 7.5\%), A763_Y764insFQEA (3/53, 5.7\%). Unique EGFRex20ins mutations detected by NGS were summarized in Fig. 2b.

EGFRex20ins tended to be exclusive with NSCLC driver genes such as EGFR mutation ERBB2, ALK, BRAF and RET mutations. The most common co-mutations were TP53 (49.1\%). Co-mutation pattern compared with

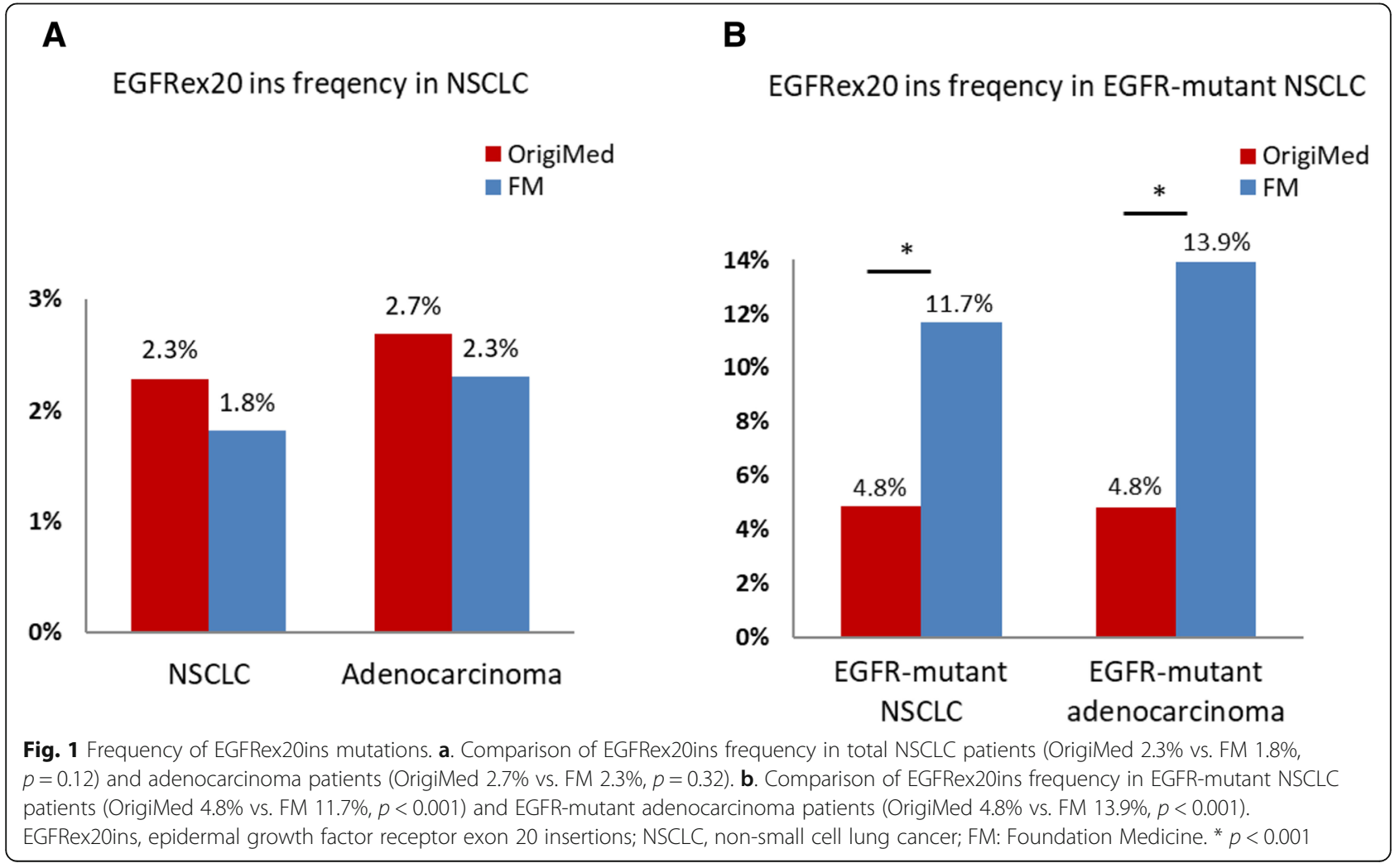


Table 1 Histologic and clinical characteristics of non-small cell lung cancer patients tested in this study

\begin{tabular}{|c|c|c|c|c|}
\hline & Adenocarcinoma & Squamous & Others $^{a}$ & Total \\
\hline Total cases & 1820 & 290 & 206 & 2316 \\
\hline Median age & $60(26-92)$ & $62(30-88)$ & $60(12-83)$ & $61(12-92)$ \\
\hline Sex, M/F & $917 / 903$ & $247 / 43$ & $144 / 62$ & $1308 / 1008$ \\
\hline EGFR mutant cases & 1021 & 11 & 63 & 1095 \\
\hline Frequency in total & $56.1 \%$ & $3.8 \%$ & $30.6 \%$ & $47.3 \%$ \\
\hline Median age & $60(26-86)$ & 64(38-89) & $57(27-74)$ & $60(26-89)$ \\
\hline Sex, M/F & $397 / 624$ & $5 / 6$ & $28 / 35$ & $430 / 665$ \\
\hline EGFRex20ins cases & 49 & 0 & 4 & 53 \\
\hline Frequency in EGFR mutant & $4.8 \%$ & 0 & $6.3 \%$ & $4.8 \%$ \\
\hline Frequency in total & $2.7 \%$ & 0 & $1.9 \%$ & $2.3 \%$ \\
\hline Median age & $57(31-85)$ & - & $56.5(49-70)$ & $57(31-85)$ \\
\hline Sex, M/F & $26 / 23$ & - & $2 / 2$ & $28 / 25$ \\
\hline
\end{tabular}

${ }^{a}$ Other pathological type in NSCLC, including adenosquamous lung cancer, NSCLC not otherwise specified, large cell lung cancer, neuroendocrine carcinoma and sarcomatoid carcinoma

$M / F$ male/female; EGFRex20ins, epidermal growth factor receptor exon 20 insertions, NSCLC non-small cell lung cancer

EGFR ex19dels, L858R, T790 M and other EGFR uncommon mutations were summarized in Additional file 1: Figure S1 and Additional file 2: Figure S2.

\section{Antitumor activity of monotherapy Osimertinib for patients with EGFRex20ins mutations}

From August 28th, 2017 to April 30th, 2018, six patients with stage IV lung adenocarcinoma bearing EGFRex20ins started osimertinib treatment. Median follow-up time was 6.2 months. Previous treatment, detailed mutation characteristics and the outcome of osimertinib are shown in Table 3. All the patients had stage IV lung adenocarcinoma and predominantly females (5/6). Before osimertinib treatment, four patients were observed metastasis in lungs and pleura. Patient 5 was diagnosed with brain metastasis and patient 6 with bone metastasis. The median age is 64 years old. Two patients received osimertinib as first line therapy and two patients had previous treatment with other EGFR TKIs. Per RECISIT 1.1, four $(67.7 \%)$ patients achieved partial response (PR) and the remaining two patients $(33.3 \%)$ obtained stable disease (SD). Median progression-free survival (PFS) was 6.2 months (95\% confidence interval 5.0-12.9 months; range 4.9-14.6 months). Treatment-related adverse events
(AEs) included diarrhea (2/6), pruritus (2/6), stomatitis (1/ 6) and nausea (1/6). No grade 3 or more AEs were documented. At data cut-off (December 1st, 2018), Two patients had sustaining disease control and remained on osimertinib treatment, while the other four patients had progressive disease (PD) ultimately.

Patient 1 had EGFR A767_V769dup mutation and received first-line therapy with osimertinib. The patient achieved PR and the PFS was 6.0 months. PR was also observed in patient 2 (EGFR S768_D770dup), with a PFS of $14.6+$ months. The patient was still on treatment at data cutoff. Patient 3 was identified with a novel EGFRex20ins mutation (EGFR N771_P772insL), which had not been reported before. The patient had SD as best response under first line osimertinib treatment and transferred to other treatment after 4.9 months due to enlarged pleural nodules. Patient 4 harbored the same EGFRex20ins mutation as that of patient 2 and attained SD with a PFS of $11.2+$ months. Both patient 2 and patient 4 remained on osimertinib treatment. Patient 5 was confirmed with multiple cerebral metastasis and experienced dizzy and vomiting when diagnosed as stage IV adenocarcinoma. The patient was treated with first line chemotherapy with the best response of PD.

Table 2 Clinical comparison of EGFR ex20ins NSCLC with EGFR

\begin{tabular}{|c|c|c|c|c|c|}
\hline & EGFR 20ins & EGFR WT & EGFR 19del & EGFR L858R & EGFR T790 M \\
\hline Median age & 57 & 61 & 59.5 & 62 & 59 \\
\hline$p$ value vs. EGFR ex20ins & & 0.399 & 0.897 & 0.206 & 0.792 \\
\hline Sex, M/F(\%M) & $28 / 25(53 \%)$ & $878 / 343(72 \%)$ & 177/259(41\%) & 130/280(32\%) & 21/37(36\%) \\
\hline$p$ value vs. EGFR ex20ins & & 0.003 & 0.088 & 0.002 & 0.078 \\
\hline
\end{tabular}

WT and EGFR mutant (19del/L858R/ T790 M) NSCLC 


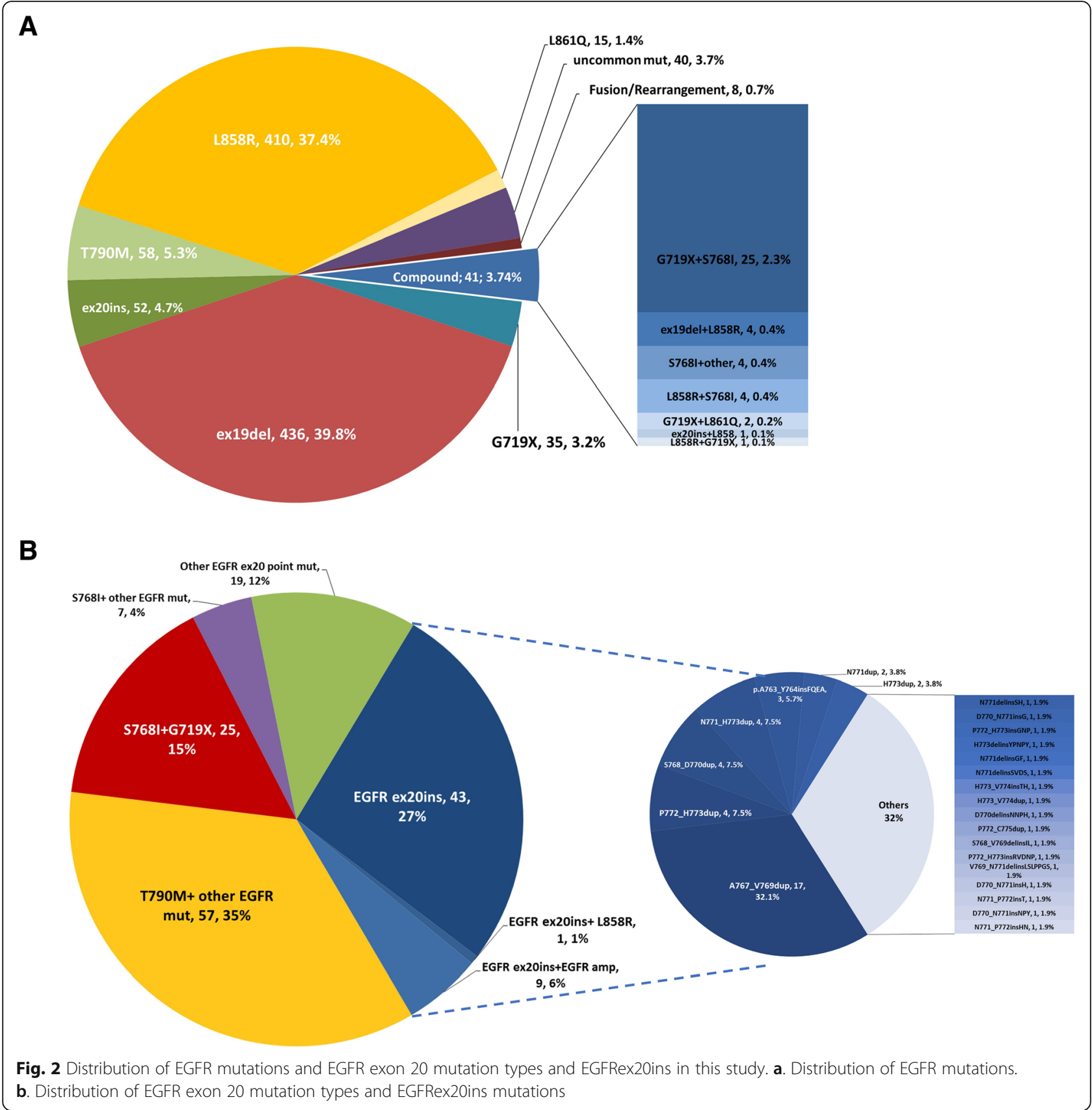

Thereafter, the patient started osimertinib and exhibited salient clinical improvement and a reduction of nearly half the tumor burden. The patient had PD finally due to new onset bone metastasis, with a PFS of 6.4 months. Patient 6 was initially diagnosed as lung adenocarcinoma harboring EGFR A763_Y764insFQEA. The patients started gefitinib treatment with a best response of PR and PFS of 9.0 months. At disease progression, the patients had rebiopsy and his tumor was found to had EGFR A763_Y764insFQEA and EGFR T790 M. Thereafter, the patient was treated with osimertinib and attained PR. The patient experienced PD eventually due to brain metastasis and achieved a PFS of 5.1 months. CT scans performed prior to (baseline) and after osimertinib treatment are demonstrated in Fig. 3. Tumor shrinkage for each patient is shown in Fig. 4.

\section{Discussion}

Based on a large-scale study including 2316 NSCLC patient, our study demonstrated the EGFRex20ins distribution in EGFR mutant Chinese patients (4.8\%), the most common EGFRex20ins mutation (A767_V769dup) and co-mutation (TP53), as well as clinical characteristics of EGFRex20ins in Chinese NSCLC patients. As for 
Table 3 Mutation characteristics and outcome of osimertinib treatment

\begin{tabular}{|c|c|c|c|c|c|c|c|}
\hline Patient & Age & Sex & $\begin{array}{l}\text { Previous Systematic } \\
\text { therapy }\end{array}$ & $\begin{array}{l}\text { Previous EGFR TKI } \\
\text { Treatment }\end{array}$ & $\begin{array}{l}\text { Mutations }{ }^{a} \text { Before } \\
\text { osimertinib (MAF) }\end{array}$ & Best Response & PFS \\
\hline 1 & $63 Y$ & $\mathrm{~F}$ & None & None & $\begin{array}{l}\text { EGFR } \\
\text { p. A767_V769dup } \\
(3.48 \%)\end{array}$ & $P R$ & $6.0 \mathrm{~m}$ \\
\hline 2 & $59 Y$ & $\mathrm{~F}$ & Yes & None & $\begin{array}{l}\text { EGFR } \\
\text { p. S768_D770dup } \\
(1.75 \%)\end{array}$ & PR Treatment ongoing & $14.6 \mathrm{~m}$ \\
\hline 3 & $69 Y$ & M & None & None & $\begin{array}{l}\text { EGFR } \\
\text { p. N771_P772insL } \\
(47 \%)\end{array}$ & SD & $4.9 \mathrm{~m}$ \\
\hline 4 & $70 Y$ & $\mathrm{~F}$ & Yes & afatinib & $\begin{array}{l}\text { EGFR } \\
\text { p. S768_D770dup } \\
(3 \%)\end{array}$ & SD Treatment ongoing & $11.2 \mathrm{~m}$ \\
\hline 5 & $63 Y$ & $\mathrm{~F}$ & Yes & None & $\begin{array}{l}\text { EGFR } \\
\text { p. D770_N771insG } \\
(24 \%)\end{array}$ & PR & $6.4 \mathrm{~m}$ \\
\hline 6 & $65 Y$ & $\mathrm{~F}$ & None & gefitinib & $\begin{array}{l}\text { EGFR } \\
\text { p. A763_Y764insFQEA (0.2\%) } \\
\text { T790 M (0.3\%) }\end{array}$ & PR & $5.1 \mathrm{~m}$ \\
\hline
\end{tabular}

${ }^{a}$ Mutations predicting EGFR TKI treatment

$T K I$ tyrosine kinase inhibitor, MAF mutation allele fraction, PFS progression free survival, $P R$ partial response, $S D$ stable disease

six EGFRex20ins positive patients with osimertinib treatment, four (67.7\%) patients achieved PR and two SD, with disease control rate $100 \%$.

To our knowledge, our study represents the largest NGS based study on Chinese EGFRex20ins mutant NSCLC patients. We found that frequency of Chinese EGFRex20ins in total NSCLC (2.3\%) was comparable with that of western groups in FM cohort [17]. Although the proportion of EGFR mutant patients was much larger in our Chinese cohort $(47.3 \%$ vs $15.5 \%)$, which consists with previous studies [1, 2], exon 20 insertions accounted for a smaller proportion compared to FM cohort in EGFR mutant NSCLC (4.8\% vs. $11.7 \%, p<0.001)$ [17]. This might mainly due to different EGFR mutation proportion between Asians and Americans, rather than sequencing technology issue as discussed in a previous study [17]. In the contrast, proportion of exon 20 insertion in EGFR- mutant patients in our study is similar to those reported in other Asian cohorts, ranging from $3.6-4 \%$ [31, 32]. These results revealed a concordance in the prevalence of EGFRex20ins between Chinese and Western populations in NSCLC.

The genetic Chinese characteristics of EGFRex20ins is similar to that of western populations, including the majority of unique mutations and the most prevalent co-mutation [17]. EGFRex20ins detected in our study also tended to be exclusive with other NSCLC oncogenic drivers including ERBB2, BRAF, ALK, KRAS and RET mutations.

Most EGFRex20ins mutations (with the exception of a few subtypes such as A763_Y764insFQEA) are associated with poor responses with the 1st and 2nd generation EGFR TKIs [19-23]. Previous clinical studies including combination therapy of afatinib plus cetuximab or monotherapy of Poziotinib have demonstrated good therapeutic efficacy in some EGFRex20ins mutations positive NSCLC patients $[24,25]$. However, the high proportion of severe AEs including skin toxicity and diarrhea of these therapies might limit their universal clinical applying in future. Several preclinical studies have proved that osimertinib was active in specific lung cancer cell lines with EGFRex20ins mutations [26-29], while the clinical activity of the 3rd generation EGFR TKIs in EGFRex20ins tumors remains unknown. Our study showed promising antitumor activity of osimertinib in NSCLC patients harboring certain EGFRex20ins mutations, with four patients attaining PR and two patients SD. Median PFS with osimertinib was 6.2 months, which was numerically higher than that with the 1st generation TKIs and afatinib [19, 22]. We also for the first time reported a novel EGFRex20ins mutation, EGFR N771_P772insL, in lung adenocarcinoma.

Despite the documented activity of osimertinib in our six patients, in vitro study still demonstrated limited osimertinib effect in several EGFRex20ins mutant cell lines [26-29]. Whether other EGFRex20ins mutant tumors could response to osimertinib warrants further study. These studies indicate EGFRex20ins is a heterogeneous group of EGFR mutation and deserves more researches to fully determine osimertinib sensitivity in different EGFRex20ins tumors.

A recent case report has showed that an advanced NSCLC patient with EGFRex20ins mutation, S768 D770dup, responded to osimertinib $160 \mathrm{mg}$ daily [33]. 


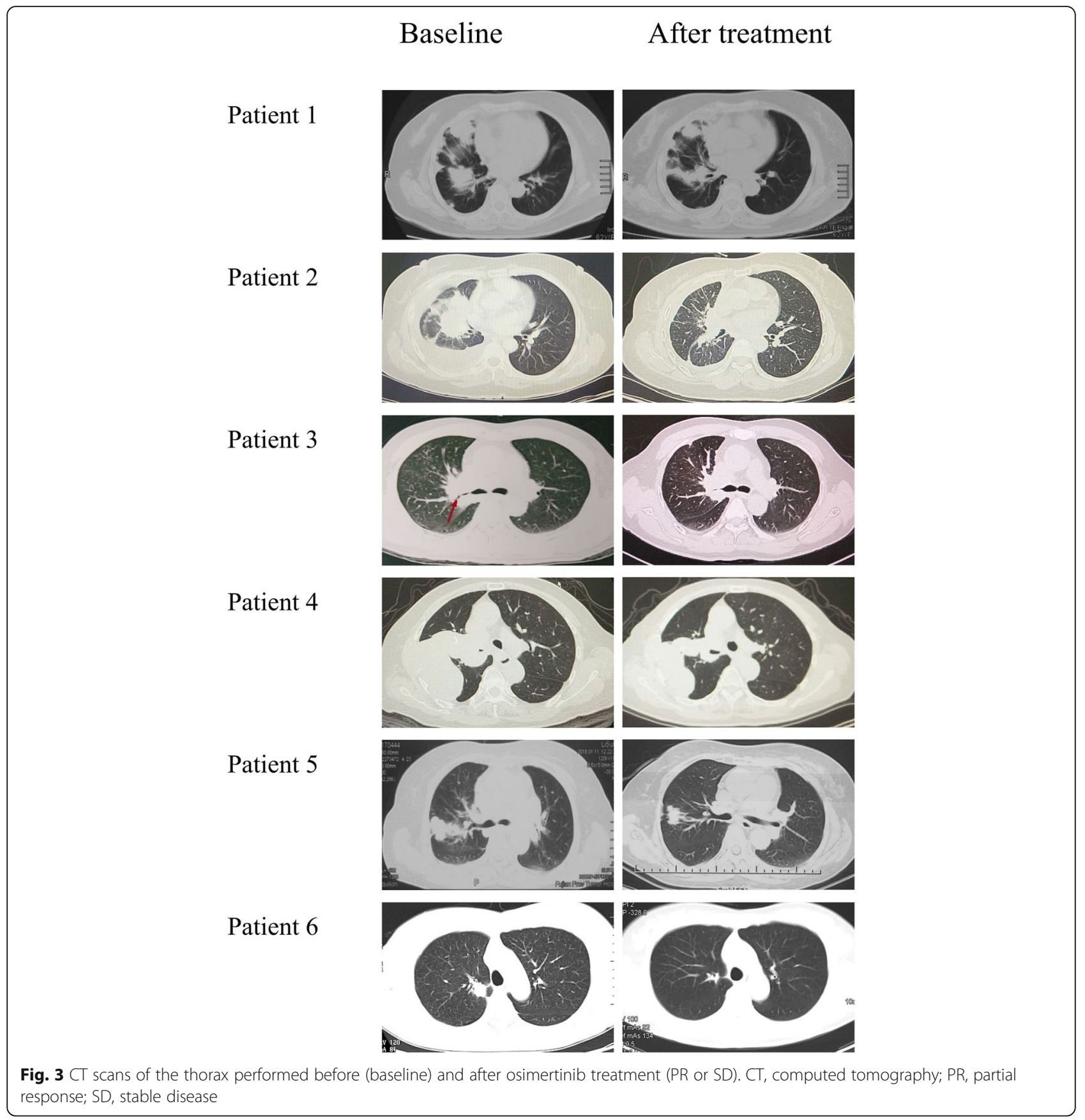

The mutation detected was the same as that of patient 2 and patient 4 , which might suggest favorable lower-dose osimertinib efficacy in tumors bearing EGFR S768_ D770dup mutation. Another case report also suggested that EGFR H773L/V774 M, an EGFR exon 20 mutation, could be suppressed by osimertinib [34], further supporting the osimertinib effect in specific EGFR exon 20 mutations.

In addition, considering that EGFR T790 M was identified in patient 6 harboring EGFR A763_Y764insFQEA after acquired resistance to gefitinib, we found that
EGFR T790 M served as a potential resistant mechanism in EGFR A763_Y764insFQEA positive NSCLC patients and occurrence of both mutations could be targeted by osimertinib. This is the first case showing that T790 M mediated the acquired resistance to gefitinib for patient with EGFR A763_Y764insFQEA and osimertinib treatment was effective for patient with both EGFR A763_Y764insFQEA and EGFR T790 M.

Still, there are several limitations in the study. Firstly, panels of NGS performed on patients are not uniform, contributing to relatively incomprehensive genetics 


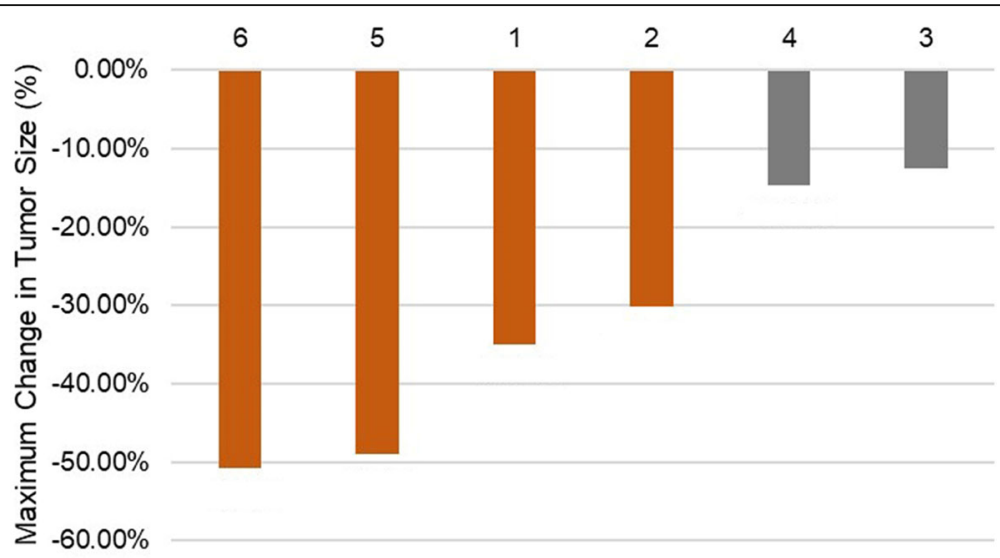

Fig. 4 Maximum change in tumor size according to Response Criteria in Solid Tumors (RECIST) 1.1. Orange grid indicates partial response and gray grid stable disease

statistics in patients with small panels. Secondly, although all six patients in our study acquired disease control, the sample size is too small to establish the therapeutic efficacy of osimertinib in patients with EGFRex20ins.

\section{Conclusions}

In summary, our study revealed no significant difference in the prevalence and genetic characteristics of EGFRex20ins between Chinese and Western populations in NSCLC. Moreover, promising antitumor activity of osimertinib was observed in specific EGFRex20ins positive NSCLC patients, more studies are urgently needed to fully determine osimertinib effect in NSCLC patients with different EGFRex20ins mutations.

\section{Additional files}

Additional file 1: Figure S1. EGFR ex20ins and co-mutation pattern. (JPG $186 \mathrm{~kb})$

Additional file 2: Figure S2. Comparison of co-mutations in EGFR ex20ins ( $n=53)$, ex19del $(n=436)$, L858R $(n=410)$, T790 M (primary and secondary mutation, $n=58$ ) and other EGFR sensitive mutations $(n=90)$. Others: Other EGFR sensitive mutations, including G719X, L861Q, S768| and compound mutations. (JPG $1798 \mathrm{~kb})$

\section{Abbreviations}

AEs: Adverse events; CT: Computed tomography; EGFRex20ins: Epidermal growth factor receptor exon 20 insertion; L858R: Leu858Arg; MRI: magnetic resonance imaging; NGS: Next generation sequencing; NOS: Non-small-cell lung cancer not otherwise specified; NSCLC: Non-small-cell lung cancer; PD: Progressive disease; PFS: Progression-free survival; PR: Partial response; RECIST: Response criteria in solid tumors; SD: Stable disease; TKIs: Tyrosine kinase inhibitors

\section{Acknowledgements}

The authors would like to thank Dr. Xiaogian Chen from OrigiMed for her excellent work in statistical support.

\section{Authors' contributions}

WF, YH, SH, ZZ, MW conceived of the study; LZ and WF designed the study; YH, SH, ZZ, MW analyzed data; JG, WW, HG, KW collected data; WF, YH, SH, ZZ wrote the manuscript. LZ, WF and SH corrected and approved the final version of the manuscript. All authors read and approved the final manuscript.

\section{Funding}

This work was financially supported by National Key R\&D Program of China (2016YFC0905500, 2016YFC0905503), Chinese National Natural Science Foundation (81772476, 81602005, 81872499, and 81702283), Science and Technology Program of Guangdong (2017B020227001). All the grand supporters have no role in the design of the study and collection, analysis, and interpretation of data and in writing the manuscript.

\section{Availability of data and materials}

The datasets used and/or analyzed during the current study are available from the corresponding author on reasonable request.

\section{Ethics approval and consent to participate}

The study was approved by the Institutional Review Board of SYSUCC and written informed consent was obtained for each patient prior to sample collection. Patients were informed that the resected specimens were stored by the hospital and potentially used for scientific research, and that their privacy would be maintained. Written consents were obtained from parents if patients were under 16 years old.

\section{Consent for publication}

Not applicable.

\section{Competing interests}

The authors declare that they have no competing interests.

\section{Author details}

${ }^{1}$ Department of Medical Oncology, State Key Laboratory of Oncology in South China, Collaborative Innovation Center for Cancer Medicine, Sun Yat-Sen University Cancer Center, Guangzhou 510060, People's Republic of China. ${ }^{2}$ Sun Yat-Sen Memorial Hospital, Sun Yat-Sen University, Guangzhou 510220, People's Republic of China. ${ }^{3}$ OrigiMed, Inc, NO.115 XinJunhuan Road, Shanghai 201114, People's Republic of China.

Received: 24 January 2019 Accepted: 11 June 2019

Published online: 17 June 2019

\section{References}

1. Kris MG, Johnson BE, Berry LD, Kwiatkowski DJ, lafrate AJ, Wistuba II, VarellaGarcia M, Franklin WA, Aronson SL, Su PF, et al. Using multiplexed assays of oncogenic drivers in lung cancers to select targeted drugs. Jama-J Am Med Assoc. 2014;311(19):1998-2006.

2. Shi Y, Li J, Zhang S, Wang M, Yang S, Li N, Wu G, Liu W, Liao G, Cai K, et al. Molecular epidemiology of EGFR mutations in Asian patients with advanced non-small-cell lung cancer of adenocarcinoma histology - mainland China subset analysis of the PIONEER study. PLoS One. 2015;10(11):e0143515. 
3. Travis WD. WHO classification of the pathology and genetics of tumors of the lung. J Thorac Oncol. 2015;10(9):S68.

4. Tsao AS, Tang XM, Sabloff B, Xiao L, Shigematsu H, Roth J, Spitz M, Hong WK, Gazdar A, Wistuba I. Clinicopathologic characteristics of the EGFR gene mutation in non-small cell lung cancer. J Thorac Oncol. 2006;1(3):231-9.

5. Lee SY, Kim MJ, Jin G, Yoo SS, Park JY, Choi JE, Jeon HS, Cho S, Lee EB, Cha $\mathrm{SI}$, et al. Somatic mutations in epidermal growth factor receptor signaling pathway genes in non-small cell lung cancers. J Thorac Oncol. 2010;5(11): 1734-40.

6. Tokumo M, Toyooka S, Kiura K, Shigematsu H, Tomii K, Aoe M, Ichimura K, Tsuda T, Yano M, Tsukuda K, et al. The relationship between epidermal growth factor receptor mutations and clinicopathologic features in nonsmall cell lung cancers. Clin Cancer Res. 2005;11(3):1167-73.

7. Rosell R, Moran T, Queralt C, Porta R, Cardenal F, Camps C, Majem M, LopezVivanco G, Isla D, Provencio M, et al. Screening for epidermal growth factor receptor mutations in lung cancer. New Engl J Med. 2009;361(10):958-67.

8. Shi Y, Au JS, Thongprasert S, Srinivasan S, Tsai CM, Khoa MT, Heeroma K, Itoh Y, Cornelio G, Yang PC. A prospective, molecular epidemiology study of EGFR mutations in Asian patients with advanced non-small-cell lung cancer of adenocarcinoma histology (PIONEER). J Thorac Oncol. 2014;9(2):154-62.

9. Mok TS, Wu YL, Thongprasert S, Yang CH, Chu DT, Saijo N, Sunpaweravong P, Han B, Margono B, Ichinose Y, et al. Gefitinib or carboplatin-paclitaxel in pulmonary adenocarcinoma. New Engl J Med. 2009;361(10):947-57.

10. Rosell R, Carcereny E, Gervais R, Vergnenegre A, Massuti B, Felip E, Palmero R, Garcia-Gomez R, Pallares C, Sanchez JM, et al. Erlotinib versus standard chemotherapy as first-line treatment for European patients with advanced EGFR mutation-positive non-small-cell lung cancer (EURTAC): a multicentre, open-label, randomised phase 3 trial. Lancet Oncol. 2012;13(3):239-46.

11. Sequist LV, Yang JC, Yamamoto N, O'Byrne K, Hirsh V, Mok T, Geater SL, Orlov S, Tsai CM, Boyer M, et al. Phase III study of afatinib or cisplatin plus pemetrexed in patients with metastatic lung adenocarcinoma with EGFR mutations. J Clin Oncol. 2013;31(27):3327-34.

12. Soria JC, Ohe Y, Vansteenkiste J, Reungwetwattana T, Chewaskulyong B, Lee $\mathrm{KH}$, Dechaphunkul A, Imamura F, Nogami N, Kurata T, et al. Osimertinib in untreated EGFR-mutated advanced non-small-cell lung Cancer. New Engl J Med. 2018;378(2):113-25.

13. Xu J, Jin B, Chu T, Dong $X$, Yang H, Zhang Y, Wu D, Lou Y, Zhang X, Wang $H$, et al. EGFR tyrosine kinase inhibitor (TKI) in patients with advanced nonsmall cell lung cancer (NSCLC) harboring uncommon EGFR mutations: a real-world study in China. Lung Cancer. 2016;96:87-92.

14. Klughammer B, Brugger W, Cappuzzo F, Ciuleanu T, Mok T, Reck M, Tan EH, Delmar P, Klingelschmitt G, Yin AY, et al. Examining treatment outcomes with Erlotinib in patients with advanced non-small cell lung cancer whose Tumors Harbor uncommon EGFR mutations. J Thorac Oncol. 2016;11(4):545-55.

15. Yasuda H, Park E, Yun CH, Sng NJ, Lucena-Araujo AR, Yeo WL, Huberman MS, Cohen DW, Nakayama S, Ishioka K, et al. Structural, biochemical, and clinical characterization of epidermal growth factor receptor (EGFR) exon 20 insertion mutations in lung cancer. Sci Transl Med. 2013;5(216):216ra177.

16. Arcila ME, Nafa K, Chaft JE, Rekhtman N, Lau C, Reva BA, Zakowski MF, Kris MG, Ladanyi M. EGFR exon 20 insertion mutations in lung adenocarcinomas: prevalence, molecular heterogeneity, and Clinicopathologic characteristics. Mol Cancer Ther. 2013;12(2):220-9.

17. Riess JW, Gandara DR, Frampton GM, Madison R, Peled N, Bufill JA, Dy G, Ignatius Ou SH, Stephens PJ, McPherson J, et al. Diverse EGFR exon 20 insertions and co-occurring molecular alterations identified by comprehensive genomic profiling of non-small cell lung Cancer. J Thorac Oncol. 2018;13(10):1560-8.

18. Oxnard GR, Lo PC, Nishino M, Dahlberg SE, Lindeman NI, Butaney M, Jackman DM, Johnson BE, Janne PA. Natural history and molecular characteristics of lung cancers harboring EGFR exon 20 insertions. J Thorac Oncol. 2013;8(2):179-84.

19. Naidoo J, Sima CS, Rodriguez K, Busby N, Nafa K, Ladanyi M, Riely GJ, Kris MG, Arcila ME, Yu HA. Epidermal growth factor receptor exon 20 insertions in advanced lung adenocarcinomas: clinical outcomes and response to erlotinib. Cancer. 2015;121(18):3212-20.

20. Yang M, Xu X, Cai J, Ning J, Wery JP, Li QX. NSCLC harboring EGFR exon-20 insertions after the regulatory C-helix of kinase domain responds poorly to known EGFR inhibitors. Int J Cancer. 2016;139(1):171-6.

21. Yasuda H, Kobayashi S, Costa DB. EGFR exon 20 insertion mutations in nonsmall-cell lung cancer: preclinical data and clinical implications. Lancet Oncol. 2012;13(1):e23-31.
22. Yang JC, Sequist LV, Geater SL, Tsai CM, Mok TS, Schuler M, Yamamoto N, Yu CJ, Ou SH, Zhou C, et al. Clinical activity of afatinib in patients with advanced non-small-cell lung cancer harbouring uncommon EGFR mutations: a combined post-hoc analysis of LUX-lung 2, LUX-lung 3, and LUX-lung 6. Lancet Oncol. 2015;16(7):830-8.

23. Keam B, Kim DW, Park JH, Lee JO, Kim TM, Lee SH, Chung DH, Heo DS. Rare and complex mutations of epidermal growth factor receptor, and efficacy of tyrosine kinase inhibitor in patients with non-small cell lung cancer. Int J Clin Oncol. 2014;19(4):594-600.

24. Robichaux JP, Elamin YY, Tan Z, Carter BW, Zhang S, Liu S, Li S, Chen T, Poteete A, Estrada-Bernal A, et al. Mechanisms and clinical activity of an EGFR and HER2 exon 20-selective kinase inhibitor in non-small cell lung cancer. Nat Med. 2018;24(5):638-46.

25. van Veggel B, de Langen AJ, Hashemi SMS, Monkhorst K, Heideman DAM, Thunnissen E, Smit EF. Afatinib and Cetuximab in four patients with EGFR exon 20 insertion-positive advanced NSCLC. J Thorac Oncol. 2018;13(8): $1222-6$.

26. Masuzawa K, Yasuda H, Hamamoto J, Nukaga S, Hirano T, Kawada I, Naoki K, Soejima K, Betsuyaku T. Characterization of the efficacies of osimertinib and nazartinib against cells expressing clinically relevant epidermal growth factor receptor mutations. Oncotarget. 2017;8(62):105479-91.

27. Hirano T, Yasuda H, Tani T, Hamamoto J, Oashi A, Ishioka K, Arai D, Nukaga S, Miyawaki M, Kawada I, et al. In vitro modeling to determine mutation specificity of EGFR tyrosine kinase inhibitors against clinically relevant EGFR mutants in non-small-cell lung cancer. Oncotarget. 2015;6(36):38789-803.

28. Floc'h N, Martin MJ, Riess JW, Orme JP, Staniszewska AD, Menard L, Cuomo ME, O'Neill DJ, Ward RA, Finlay MRV, et al. Antitumor activity of Osimertinib, an irreversible mutant-selective EGFR tyrosine kinase inhibitor, in NSCLC harboring EGFR exon 20 insertions. Mol Cancer Ther. 2018;17(5):885-96.

29. Ruan Z, Kannan N. Altered conformational landscape and dimerization dependency underpins the activation of EGFR by alphaC-beta4 loop insertion mutations. Proc Natl Acad Sci U S A. 2018;115(35):E8162-71.

30. Eisenhauer EA, Therasse P, Bogaerts J, Schwartz LH, Sargent D, Ford R, Dancey J, Arbuck S, Gwyther S, Mooney M, et al. New response evaluation criteria in solid tumours: revised RECIST guideline (version 1.1). Eur J Cancer. 2009;45(2):228-47.

31. Tu HY, Ke EE, Yang JJ, Sun YL, Yan HH, Zheng MY, Bai XY, Wang Z, Su J, Chen ZH, et al. A comprehensive review of uncommon EGFR mutations in patients with non-small cell lung cancer. Lung Cancer. 2017;114:96-102.

32. Shen YC, Tseng GC, Tu CY, Chen WC, Liao WC, Chen WC, Li CH, Chen HJ, Hsia TC. Comparing the effects of afatinib with gefitinib or Erlotinib in patients with advanced-stage lung adenocarcinoma harboring non-classical epidermal growth factor receptor mutations. Lung Cancer. 2017;110:56-62.

33. Piotrowska Z, Fintelmann FJ, Sequist LV, Jahagirdar B. Response to Osimertinib in an EGFR exon 20 insertion-positive lung adenocarcinoma. J Thorac Oncol. 2018;13(10):e204-6.

34. Yang M, Tong X, Xu X, Zheng E, Ni J, Li J, Yan J, Shao YW, Zhao G. Case report: Osimertinib achieved remarkable and sustained disease control in an advanced non-small-cell lung cancer harboring EGFR H773LN774M mutation complex. Lung Cancer. 2018;121:1-4.

\section{Publisher's Note}

Springer Nature remains neutral with regard to jurisdictional claims in published maps and institutional affiliations.

Ready to submit your research? Choose BMC and benefit from:

- fast, convenient online submission

- thorough peer review by experienced researchers in your field

- rapid publication on acceptance

- support for research data, including large and complex data types

- gold Open Access which fosters wider collaboration and increased citations

- maximum visibility for your research: over $100 \mathrm{M}$ website views per year

At BMC, research is always in progress.

Learn more biomedcentral.com/submission 